\title{
CONTINUOUS MICROMIXER WITH PULSATILE MICROPUMPS
}

\author{
Ajay A. Deshmukh, Dorian Liepmann and Albert P. Pisano \\ Berkeley Sensor and Actuator Center \\ University of California, Berkeley \\ 497 Cory Hall, Berkeley, CA 94720
}

\begin{abstract}
A continuous microfluidic mixer utilizing pulsatile flow micropumps has been created using an SOI process. While individual MEMS mixers, pumps, and valves have been developed, this work presents an integrated, planar system utilizing novel mixing, valving, and bonding processes in order to deliver well mixed fluids at controllable flow rates.
\end{abstract}

\section{INTRODUCTION}

At small scales, using conventional mixing methods (such as turbulence) to mix fluids is almost impossible since even diffusion can be too slow. However effective mixing is stll required for many microfluidic devices, with applications ranging from drug delivery to chemical analysis. Towards this need a new process for delivering well-mixed fluids from a MEMS device has been developed.

\section{THEORY}

Pulsatile flow can create a greatly lengthened fluid interface and much faster mixing. This process has been modeled numerically with water (white) and water with glucose (black) flowing through a $5 \mathrm{~mm}$ long, 400 micron wide channel at an average speed of $6 \mathrm{~mm}$ per second $(\operatorname{Re}=2.4)$. Figure la shows the result of steady flow with diffusion; very little mixing occurs by the end. (Modeling done using the CFDRC's code, CFD$\mathrm{ACE}$; the pictures have been stretched in the vertical direction.)

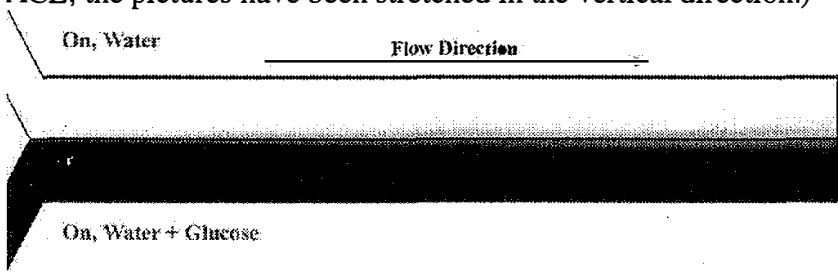

Figure 1a. Steady flow

At low Reynolds numbers, if one stream is stopped, the other stream will intrude into the other half of the main channel. In Fig. lb, the top stream is turned off for $1 / 6 \mathrm{sec}$ while the dark fluid from the bottom continues flowing.

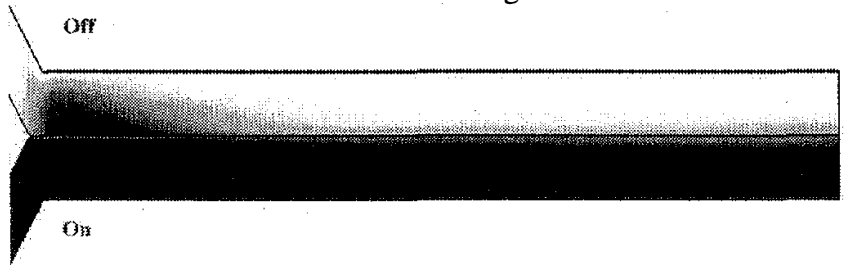

Figure 1b. The top stream is stopped for $1 / 6 \mathrm{sec}$.

As the fluid moves downstream, the velocity gradient in the channel will distort the intrusion and lengthen the interface even further. In Fig. 1c, the top stream is turned on and pushes the first intrusion downstream. Since the fluid travels faster at the centerline than at the edge, the dark intrusion is further distorted by the velocity profile. This distortion appears as a crescent shaped interface. The velocity profile is similar to, although not exactly like, the parabolic profile obtained from Poiseuille flow.

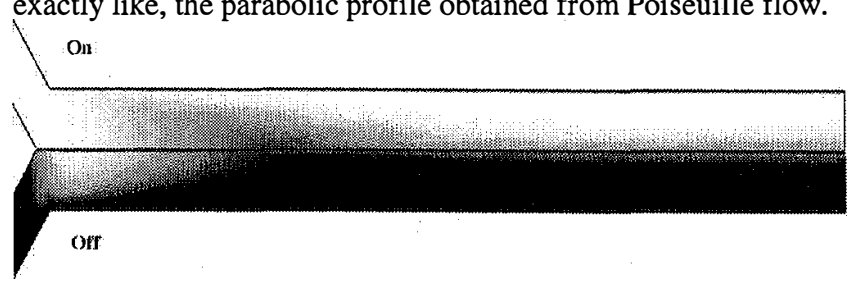

Figure 1c. The bottom stream is stopped

Fig. 1d is a picture of the flow when each stream has been turned on and off, out of phase with the other, at $3 \mathrm{~Hz}$. The fluid mixes within a few channel diameters from the fluid junction. While not a steady state solution, this scenario will reoccur every $1 / 3 \mathrm{sec}$. The average speed of the exiting fluid is $3.5 \mathrm{~mm} / \mathrm{sec}$. on

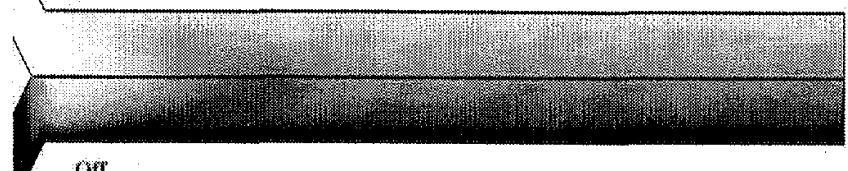

Figure 1d. Well-mixed fluid after many pulses

This novel approach to mixing allows for precise outlet composition via control of inlet stream duty-cycles and frequencies as well as continuous fluid delivery; these advantages are not possible using batch processes (e.g. Evans[1]) or passive mixers (e.g. Jaeggi[2]).

Pulsatile flow control is absolutely required for this mixing process. For the current system, positive displacement pumps have been developed. A schematic of the device is shown in Fig. 2a. Fluid 1 (white) is pumped into the mixing channel while Fluid 2 (black) is drawn in from the inlet.

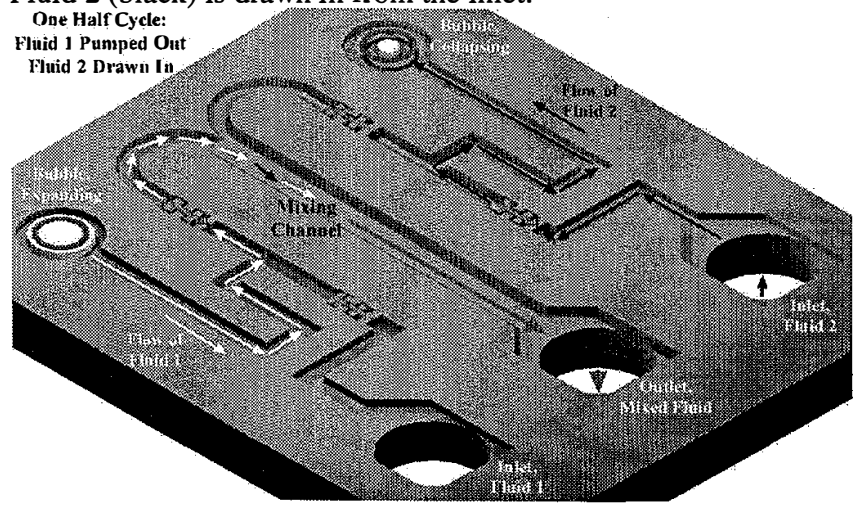

Figure 2a. Schematic of a Micromixer

The pumps consist of a bubble chamber and two check valves. When a bubble is created, it acts as a piston and drives fluid out; the check valves allow fluid to exit only in the output direction. When the bubble collapses, fluid is drawn in, but can 
only enter from the inlet side. By cycling bubble generation and collapse, a net pumping action occurs. A close-up of this design is shown in Fig. 2b. (A very different piston and check valve pump is also shown in Bardell[3]).

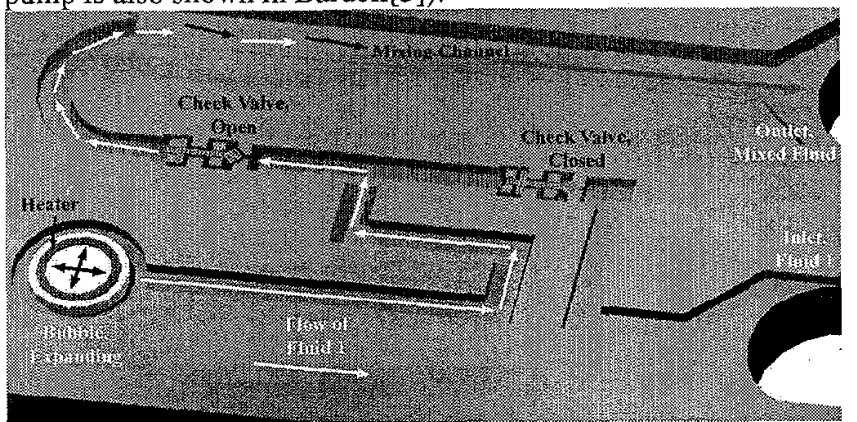

Figure $2 b$. Close-up of a pump

Steam bubbles can be generated using heat. When the heat is removed, the steam will re-condense and the bubble will collapse. In many cases, boiling the delivered fluid is undesirable. While boiling some of the fluid is necessary, a long channel connecting the bubble chamber to the main flow channel will isolate the working fluid from the delivered fluid.

\section{DESIGN}

The micromixer has been fabricated using SOI and quartz wafers bonded together. Bubbles are created by polysilicon resistors on quartz which act as heaters. In Fig. $3 \mathrm{a}$ is shown an early version of a heater and bubble chamber: the diameter of the bubble chamber is 800 microns. In Fig. $3 \mathrm{~b}$ is shown a close-up of a new heater design that is more robust.
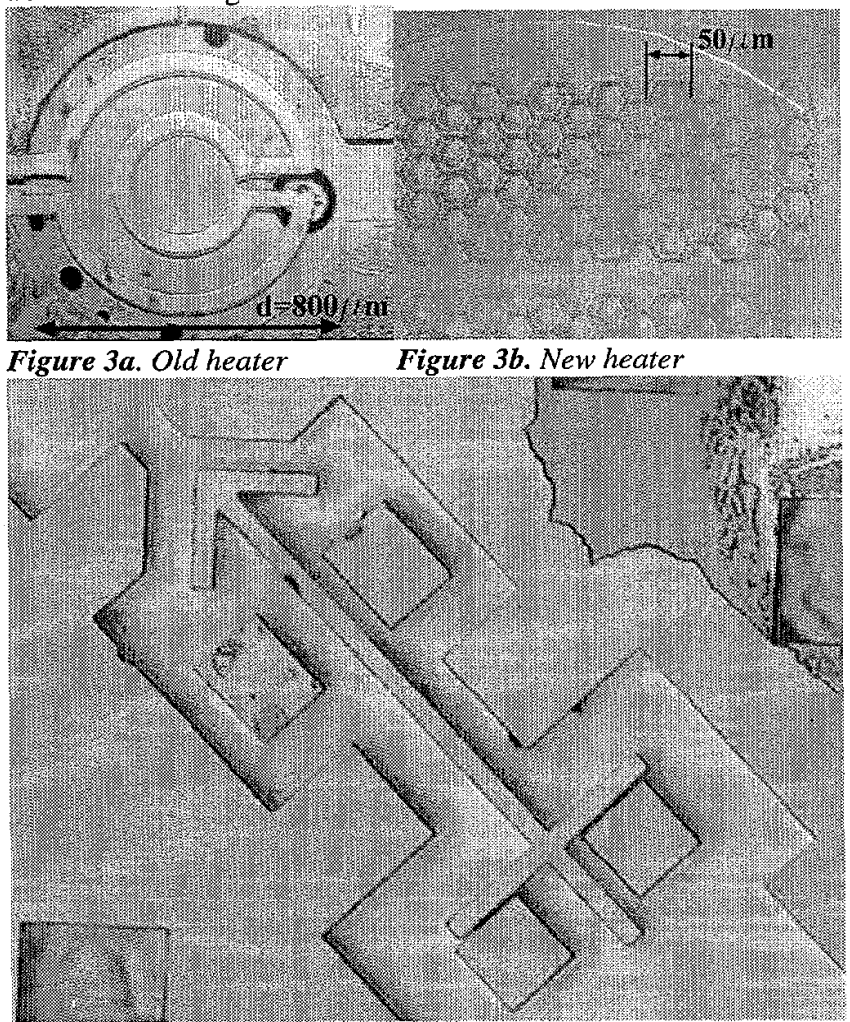

Figure 4. A check valve

The check valves are a new design consisting of free-floating silicon pieces that are actuated by viscous drag. The check valve in Fig. 4 is free to move back and forth; flow to the lower right will continue unimpeded, whereas flow to the top left will close the valve. Note that these valves operate in the plane of the wafer; most previously disclosed MEMS valves (e.g. Wroblewski[4]) control flow that is out of the plane of the substrate. The channel width is 200 microns; the valve body length is 450 microns.

\section{FABRICATION}

The mixer is made of Silicon on Insulator (SOI) and quartz dice. The SOI wafer has a 2 micron thick buried oxide layer and a 75 micron device layer. Two sequential Deep Reactive Ion Etches (DRIE) are performed; the first going all the way through the wafer to form throughholes, while the second going only through the device layer to create the channels. A 1.3 micron thick wet oxide layer provides the mask for the second etch while a layer of 9.5 micron thick photoresist over the oxide serves as the mask for the first etch. After the DRIE processes, the valves are almost completely freed from the substrate in HF. They are then placed in $\mathrm{H}_{2} \mathrm{O}_{2}$ to gencrate a thin oxide layer on the bottom of the valve. Afterwards, a probe tip is used to gently free the valves.

The quartz wafer has doped polysilicon and sputtered aluminum applied, both of around 0.3 micron thickness. The layers, after being patterned into heaters, are passivated with silicon oxide and/or silicon nitride, leaving openings for the electrical connections. A novel process for bonding is used to attach the quartz cover plate. A low viscosity epoxy (Epotek 301) is spun on at $10,000 \mathrm{rpm}$, with tape applied to the electrical contacts to prevent epoxy from covering them. The two dies are then 'flip-chipped', with pressures of between $100-300 \mathrm{kPa}$ per die. The epoxy bonds and seals the dies but also traps the valve bodies. In Fig. 5a, the covering die has been bonded on top and an epoxy layer fills the gap between the two surfaces.
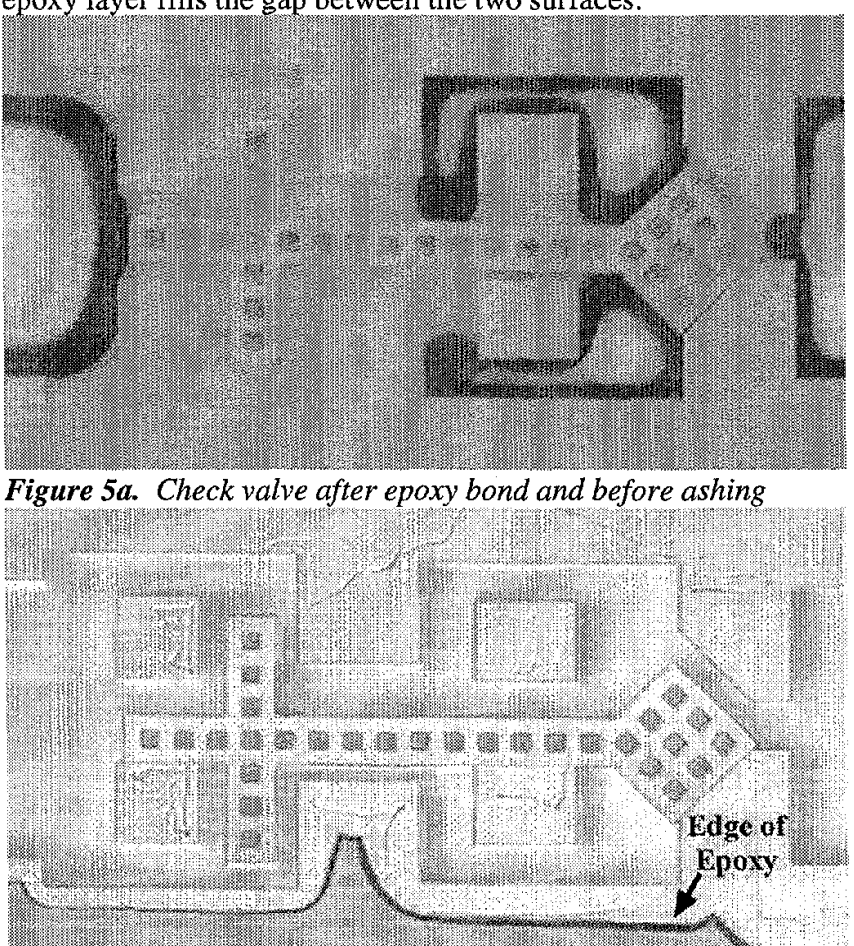

Figure 5b. Valve from Fig. $9 a$ after ashing (note that it has moved) 
An oxygen plasma ( $200 \mathrm{~W}$ in 600 mtorr of $\mathrm{O}_{2}$ ) is used to remove the epoxy in the fluid channels. Throughholes allow the plasma to access the fluid channels. The oxygen first removes the epoxy on the top of the channels. The plasma then ashes the epoxy in between the silicon and the quartz. It then moves slowly outward from the channel. The plasma etching is timed to free the valves without penetrating far from the channel edge. The valves are freed, but sufficient epoxy remains outside the channels to bond and seal the device.

In Fig. 5b the edge of the epoxy layer can be seen. The epoxy has been etched farther above the channel than below it, possibly due to non-uniformity in the epoxy layer. Once the valves are free, the devices are placed in an $\mathrm{H}_{2} \mathrm{O}_{2}$ and surfactant ultrasound bath in order to create an oxide layer surrounding the valve bodies. Polyamide tubing is then epoxied into the throughholes.

A descriptive cross section is shown in Fig. 6; it does not correspond to any actual cross section in the device but is a combination of the heater and valve structures.

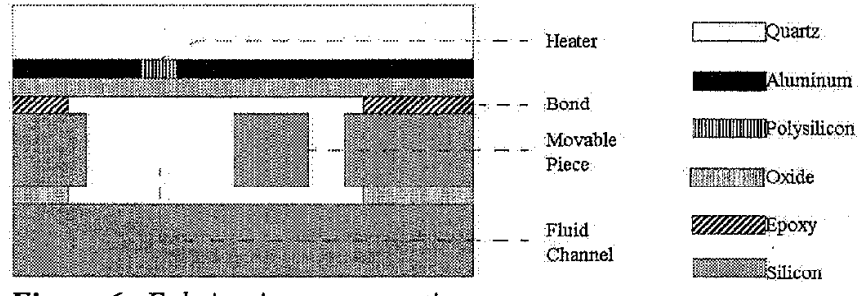

Figure 6. Fabrication cross section

\section{EXPERIMENTAL DETALS}

In the following sequence of photographs, a bubble in the fluid can be used to visualize fluid flow.

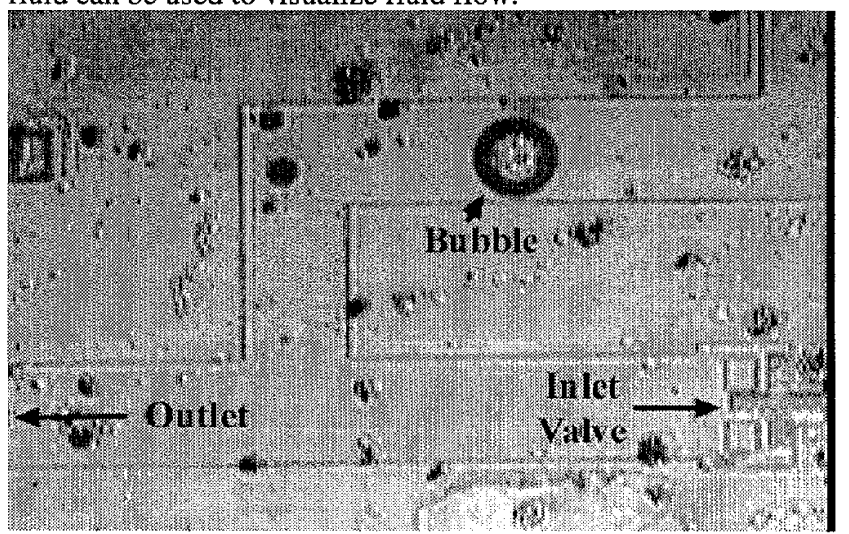

Figure 7a. Flow channel with a bubble inside

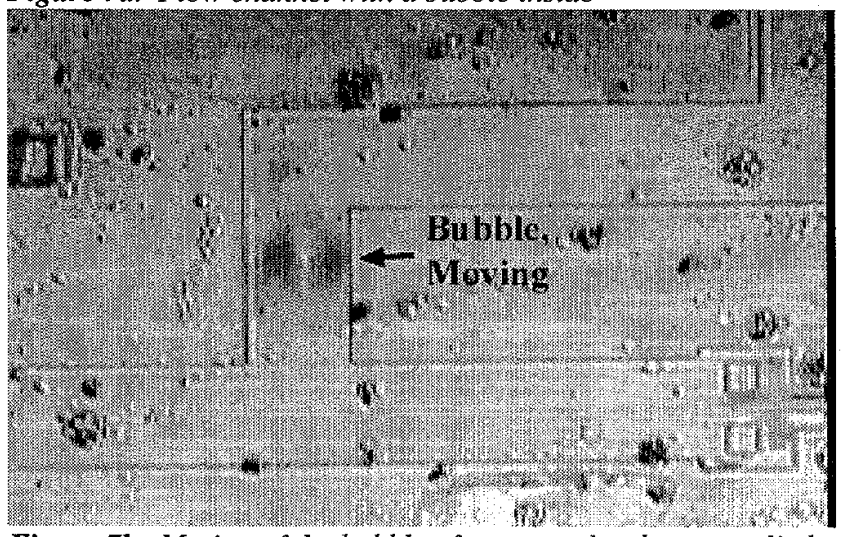

Figure $7 b$. Motion of the bubble after power has been supplied

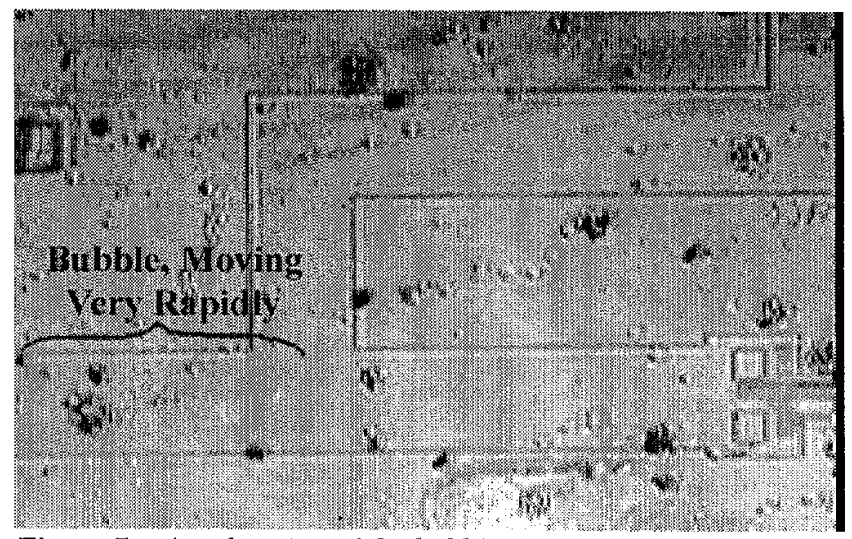

Figure 7c. Acceleration of the bubble as power is maintained

The bubble is originally stationary, but when the heater is activated, the fluid starts moving and travels through the outlet. The velocity of the bubble in Fig. $7 \mathrm{c}$ is approximately $15 \mathrm{~mm} / \mathrm{sec}$. This represents a peak and not an average velocity for the pump. Figures $8 \mathrm{a}$ and $\mathrm{b}$ isolate the outlet check valve.

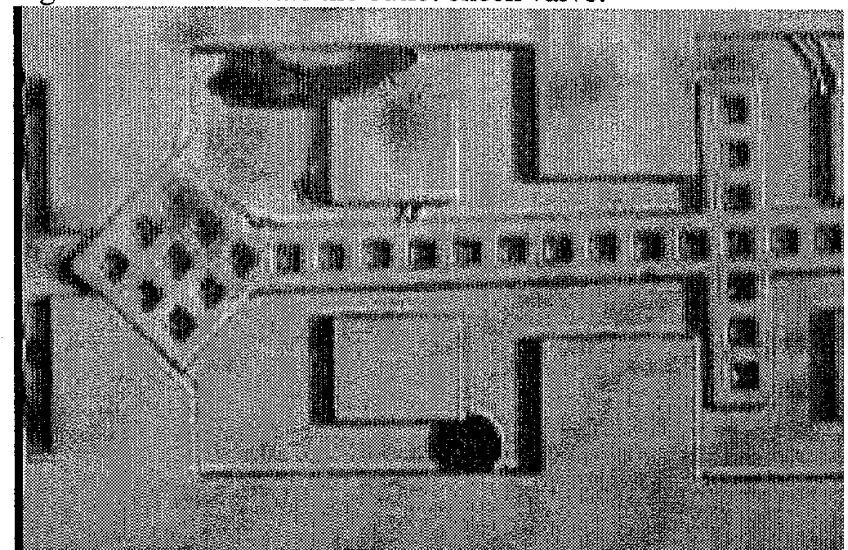

Figure 8a. Outlet valve with power off

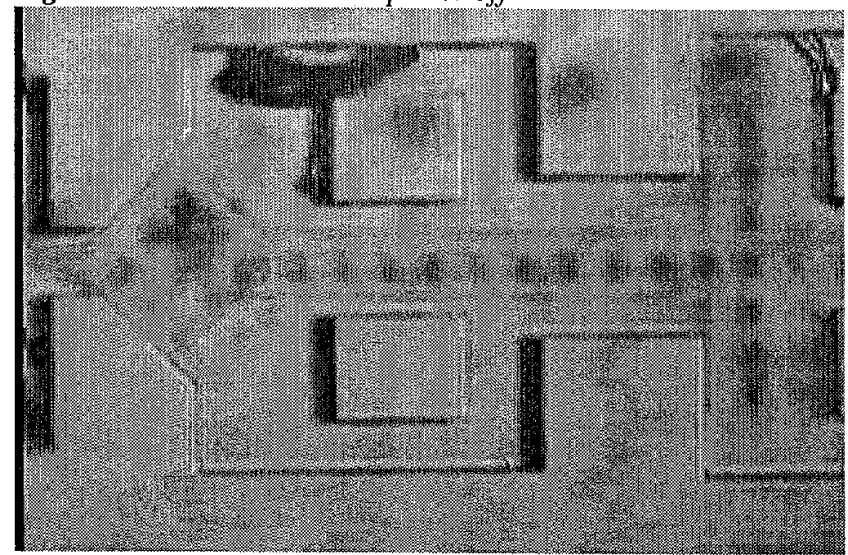

Figure 8b. Valve with power on

In Fig. 8b, the valve vibrates instead of simply staying open. This vibration is caused by the existence of a secondary oscillation in the flow. This oscillation is due to an instability that causes the bubble to fluctuate in size when the power is on. This secondary oscillation not only causes the valve body to move as seen but also actually provides additional pumping force.

Flow rates of around $5 \mathrm{microliters} / \mathrm{min}$ have been attained using pumping frequencies of approximately $0.5 \mathrm{~Hz}$. The pump heaters require 1 to 4 Watts to thermally generate a bubble, but the average power is lower. While this is higher than desired, several optimisation strategies to lower power use are possible. 
For example, if the desired flow rate is low, the pumps can be operated more slowly, with the heaters off longer than on. Better thermal isolation of the heater and bubble chamber will reduce heat loss to the substrate. Electrolysis bubbles might be usable as well; see Papavasiliou[5].

Mixing has been demonstrated with water containing either fluorescein (white) and food coloring (black). In Fig. 9 is shown four frames from a video recording taken $0.1 \mathrm{sec}$ apart. The fluid is being pumped. The light colored fluid enters the mixing channel and curls into the lower half. The large dark bulge is consequently forced downstream. The interface between the two fluids stretches and distorts as the flow develops further. This distortion is highlighted in Fig. 10. The boundary between the two fluids becomes longer, more crescent shaped, and less distinct towards the right (outlet) end of the channel. Mixing takes place rapidly because of the increase in the interfacial area across which diffusion occurs.

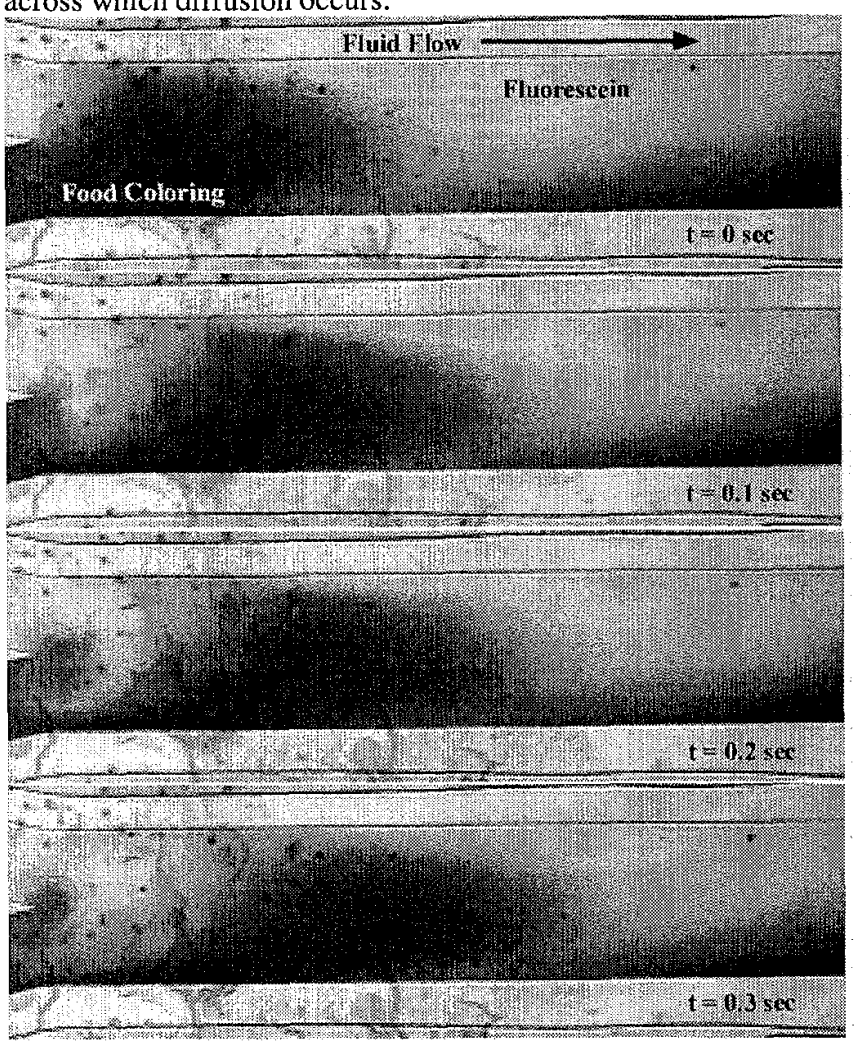

Figure 9. Mixing channel (4 frames)

The channel is 400 microns wide and a little more than $2 \mathrm{~mm}$ of length is visible. The flow rate here is estimated at about 1.5 microliters/min; the Reynolds number is approximately 0.06 .

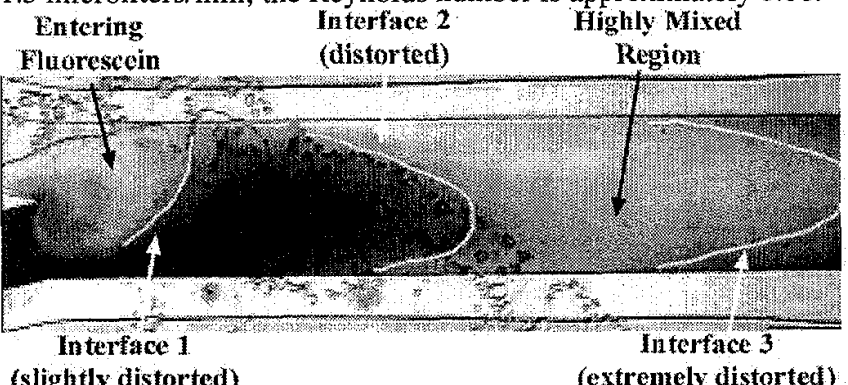

(slightly distorted)

(extremely distorted)

\section{CONCLUSIONS}

An integrated microfluidic system for mixing has been developed. This system includes several novel fluid control components: free floating micro check valves, crack resistant thermal actuators and planar positive displacement micropumps. In addition, these components have been integrated into a single MEMS system capable of mixing and delivering any two fluids with arbitrary composition.

This system has demonstrated that unsteady forcing of a twodimensional flow can efficiently mix two fluids in a continuous process (c.f. Evans [1]). While much work remains to be done to improve reliability and efficiency, a practical MEMS microfluidic mixer has been created. Moreover, the mixer is planar, which allows for easy integration with other microfluidic components.

\section{ACKNOWLEDGEMENTS}

The authors would like to thank Alex Papavasiliou and John Evans for their tremendous assistance with fabrication and design. The support of DARPA through its MicroFLUMES program (Contract F33615-97-1-3730) under the leadership of Dr. Abe Lee is also greatly appreciated.

\section{REFERENCES}

1. Evans, J.D., Liepmann, D., Pisano, A. P., "Planar Laminar Mixer", MEMS97, Nagoya, Japan, (1997), pp 96-101.

2. Jaeggi, D., Gray, B. L., Mourlas, N. J., van Drieënhuizen, B. P., Williams, K. R., Maluf, N. I., and Kovacs, G. T. A., "Novel Interconnection Technologies for Integrated Microfluidic Systems," Proceedings of the Solid-State Sensor and Actuator Workshop, Hilton Head, SC, (1998), pp. 112-115.

3. Bardell, R., Sharma, N.R., Forster, F.K., Afromowitz M.A., and Penny, $R$. "Designing high-performance micro-pumps based on no-moving-parts valves," Micro-Electrical-Mechanical Systems (MEMS), 1997 ASME International Mechanical Engineering Congress and Exposition, Dallas, TX, DSC-Vol. 234, (1997), pp. 47-53.

4. Wroblewski, D. E., Horenstein, M., Vandclli, N., Velonis, M., Bifano, T. "MEMS Micro-Valve Arrays for Fluidic Control" Micro-Electrical-Mechanical Systems (MEMS), C.J. Kim, et al., eds., 1998 ASME International Mechanical Engineering Congress and Exposition, Anaheim, CA, DSC-Vol. 66, (1998), pp. 145-151. 5. Papavasiliou, A., Liepmann, D., Pisano, A. P., "Fabrication of Free Floating Silicon Gate Valve," Proceedings of the ASME MEMS Division, 1999 IMECE, vol: 1 pp. 435-440. 